\title{
Using DAM to drive workflow: A concept for transforming information into knowledge
}

\begin{abstract}
Bob Schaffel
is an industry consultant and former senior product manager, Adobe Systems. Bob Schaffel has 15 years of experience envisioning, defining and successfully introducing new products and relevant technology to target communities, and is providing strategic and business analysis to technology companies. He has specialized on products related to workflow and collaboration, and focused on turning information and metadata into knowledge-based solutions. Bob was most recently senior product manager at Adobe Systems Inc., where he created, designed and brought to market Adobe Version Cue, the digital asset management feature of Adobe Creative Suite. He also product managed XMP (Extensible Metadata Platform) and was the product manager involved in the creation of Job Definition Format (JDF). Prior to this, Bob was manager of emerging technologies for RR Donnelley \& Sons, where his responsibilities involved strategic and operational technology implementation for the production of electronic and printed publications. Bob has spoken frequently in the US and internationally on technology processes and strategies, and has authored many articles about publishing workflows and technology.
\end{abstract}

Keywords: workflow management, process management, metadata, XMP, knowledge management

Abstract Companies are asking their process management solutions to do more to monitor and optimize end-to-end workflow. Individual solutions, such as DAM, that are targeted at solving particular business problems are great at dealing with the complexity of those problems within their own domain. They need to contribute to a process management solution that can not only be monitored but that can also be used to handle the cross-domain information that is the foundation of workflow. This paper suggests a common definition of workflow along with a proposal for a model for workflow management that is based on using cross-domain metadata to create a knowledge-based solution to track and improve process management. Journal of Digital Asset Management (2006) 2, 129-133. doi:10.1057/palgrave.dam.3650022

\section{DAM IS NOT JUST FOR BREAKFAST ANY MORE}

DAM is growing up. That is to say, it's not just that the systems are becoming more robust, reliable and scalable. Companies are asking DAM systems to do a lot more than manage the locating, accessing and (re-)distribution of assets. The latest area of expanded functionality is workflow, or rather, the monitoring and optimization of workflow based on an asset-centric view of the state and status of business and production processes.

ob Schaffel 1220, Brahms Terrace, $\# 300$,

Fremont, CA 94538

USA

Tel: +1510 2994992

E-mail:

schaffel@ catenary.org

\section{WORKFLOW MANAGEMENT CREATES KNOWLEDGE}

Let us examine this from a different perspective. Workflow management is not just an expansion of functionality. It could be argued that workflow management is a fundamental goal of the solutions we are implementing, and that DAM, enterprise content management, Digital Rights Management (DRM), business process management are really just its particular specializations arising from the need to solve particular business problems. We must then design and install systems that model and understand workflow by handling crossdomain information. Doing this not only enables us to implement expanded functionality (exposing relevant information) but we're actually building a scalable infrastructure that turns related metadata, content, assets, transactions and other business-related information into knowledge - knowledge that drives a host of improvements in service of increased profitability. 


\section{WORKFLOW IS WHAT WORKFLOW DOES?}

To understand the contribution DAM can make to workflow, we must arrive at a broader definition of "workflow" than is customarily used. Well, that's actually one of the problems: there is no customary definition of workflow. Depending on where they sit in a company's hierarchy, different people will model workflow on the basis of the view from their own seats. Here are just three of many different notions of workflow.

1. A studio manager may need to track the progress of the reviews of design concepts for an advertising campaign. As part of readying magazine sections for release to production, a production manager may need to monitor the status of photography and artwork as they move from research to art direction, to selection for publication to final review and approval. These are views of microprocesses that help validate status. Modeling them can help uncover costly inefficiencies.

2. Program managers often model workflow as a sequence of high-level activities to propose and test scheduling assumptions, allocate resources and plan fallbacks in the event of unforeseen difficulties. Creating a new product identity is an example and a flowchart would include a number of different phases, including, for example, research, concept development and prototyping to development, testing and release to market. Such macroplanning provides a way to scope the overall effort, plan the course of third-party interaction and determine the business metrics under which the project will proceed (among a host of other important areas).

3. IT managers are now being asked to tie the information from disparate legacy management solutions to in-process workflow management. This means not only accessing relevant information and metadata from disparate systems but managing the implied and explicit relationships between the information about stored entities that have always existed but been costly or impossible to reveal. Using DRM metadata to provide semi-automated gatekeeping to prevent the inadvertent (but possibly costly) misuse of assets stored in a DAM is one example of the need to confederate the information and processes across disparate domains.

Each of these points of view solves a particular business problem but engenders a solution that creates its own "silo" of information and metadata because each solution regulates and reveals the condition of only one part of the workflow. Without a different model for workflow that envisages cross-domain information coming together, it is not only an uphill battle to get insight into the status of what his happening across the domains, but is impossible to get at the knowledge that generates process improvement as well.

\section{GETTING AT A COMMON VIEW OF WORKFLOW}

Having cross-domain information available that will produce this knowledge implies being able to handle more information - more metadata. This has driven quite a bit of discussion in the community about approaches to rationalizing the metadata schemas. But, to affect workflow and to effect workflow there is more that is required than taxonomies to describe assets or content. Descriptors are required for the status of the entities and of equivalent importance is the model for the state of the processes themselves.

To create knowledge, an additional dimension of information must be revealed and managed: the relationships between entities. It is the ability not only to reveal information and display it but to correlate it into relationships between the entities that effects workflow as entities are passed from one phase of the work to the next, and as these entities relate differently in the context of the next phase. Tracking the relationships and understanding their context is what differentiates next wave systems.

Thus, a definition of workflow that might be more common to process management across domains would be: the guidance (metadata) required to reveal the relationships between the entities so that processes can be monitored and controlled for maximum efficiency. It is the 
discovery of these relationships and the maintenance of their context that allows the walls of the silos to be lowered. It's true that this sets the bar higher, but it also has enormous potential to deliver the knowledge required not just to monitor workflow but improve it.

\section{THE CONTEXT OF WORKFLOW}

The notion of relationships between entities isn't hard to understand. We're used to seeing evidence of them in each of the specific workflow silos mentioned above. But we need a way to visualize the context of workflow as well. One useful starting point is a threedimensional (3D) scatter chart. One of the benefits of a scatter chart is the ability to visualize clusters of information. The context of the view of the clusters is dependent on the viewer's relationship to the $3 \mathrm{D}$ chart. As the viewer "walks around" the chart figuratively (or more aptly, as the chart is rotated), the context of understanding changes as some hidden clusters of data come into view, or as clusters are seen to be oriented closer or farther from each other.

How does this relate to workflow? We usually think of process as a linear sequence of events. It's natural because time after all is linear. However, where time can only move in one direction. Unexpected events often turn the clock backward on workflow. For example, last minute changes in magazine copy based on current events may send the workflow back from the printing press all the way to editorial for the updates. Because of the need to cope with what is, in effect, infinite variability, workflow is more aptly modeled as clusters of processes. Within each cluster, events may proceed more or less linearly. But the progress of work from cluster to cluster may indeed not be linear at all.

Systems that model process linearly have difficulty coping with the unexpected events that affect daily workflow. Additionally, within each cluster, the entities (assets, content, etc) spawn temporal relationships that come and go as different combinations of assets move through the process asynchronously. The essence of workflow is monitoring and tracking the dynamic and volatile relationships within a process, and correlating the relationships between the clusters. This is the essence of context.

\section{PERSISTENCE OF CONTEXT}

We monitor the information within the context that is relevant to our job responsibilities. "Walking around" the figurative scatter chart gives us the opportunity to choose the view most relevant to our needs or function. What's interesting about this model is that the view from each context is persistent... that is, not only does the view have validity from the viewing position, but the relationships between the clusters is persistent and can be evaluated from each different viewing position.

Additionally, if we can return to alternative contexts without having to start from scratch to build the relationships within and among the clusters, we can begin to extract understanding of the relationships that gives us the knowledge we need to improve. We can make changes with a cluster and see the effect on other clusters, or we can change the relationships between the clusters themselves and monitor what happens to the processes within. Context is created when these relationships are correlated. Conversely, choosing a context can drive which relationships are revealed. This is what distinguishes a workflow management system from "simple" DAM very powerful.

\section{DAM AS A CROSS-DOMAIN ENVIRONMENT}

In similar fashion to workflow models that create silos, most DAM systems themselves are in their own conceptual silos — and thus also, by intent, focused on a particular part of the process chain. The archetypical DAM system is a distribution DAM. It collects the creations of some and makes them available, usually in final form, to others. The key activities are preparation for access, storage and retrieval. Some people interact with it by contributing assets to one or more repositories, and others interact by searching to discover what is available, validating that an asset is the "right one" and retrieving it from the DAM. The assets can then be put to a myriad of other uses.

Although an archive is usually thought of as a kind of warehousing, it too is a form of DAM. 
There have been a number of models for managing assets in online, offline and near-line archives. These won't be discussed in this article.

The concepts of distribution DAM and archive DAM are more closely related to warehousing than process monitoring and improvement. But another model is emerging whose goal is to go beyond storage, access, search and retrieval. This is the in-process DAM that adds the notion that the solution can monitor information about the condition of the processes as well as the assets themselves. An expanded range of information tracks such things as status, version, permissions, rights, suitability, etc on the basis of the context an asset or set of assets are in. The ability to correlate cross-domain metadata in the context of the workflow is what's new about this kind of DAM.

\section{INTELLIGENT MEDIA CONTRIBUTE TO INFORMED WORKFLOW}

Until recently, the system has been the ruler of what an asset can and cannot contribute to the knowledge base that makes workflow management possible. But with the advent of XMP (Extensible Metadata Platform) from Adobe, assets no longer are enslaved by the system. XMP is a framework for embedding metadata into assets directly rather than housing the information solely in a database associated with the solution. This turns the assets into intelligent media that contain rich metadata that are carried from one context to the next by the asset. The information is preserved across transformations of the asset and is easily accessible.

Asset metadata make a system smarter by completing the system metadata picture. A person viewing the information within a process cluster has access to not only the most relevant data for that process and in that context, but can also understand better how that information relates to other clusters of the workflow whether seen in the same or different contexts.

A DAM system modeled in this way can begin to treat the knowledge as an asset as well as the media itself. With cross-domain, relevant information available on demand within the contexts of the workflow clusters, the knowledge enables informed human decisions about the workflow and can drive a new level of automation. The expanded information can also be culled historically for the analysis of the performance of the solution.

\section{ACTIVE HARVESTING OF METADATA}

Once DAM solutions are modeled this way, another aspect that can be improved is in the realm of how active the solution itself is. Since the assets themselves are more intelligent, the system has a lot more information that it can choose from because the assets have been imbued with it. Thus, rather than people having to supply metadata manually from one context to a different one, the asset can do the work. This transforms DAM from being a passive collector of people-supplied metadata into an active harvester of the information. The burden is eased for the users to constantly be supplying information manually that is otherwise available in the other contexts.

\section{INTEGRATING DAM INTO WORKFLOW}

There is one additional contribution that intelligent assets within a DAM can do to help create informed workflow. An intelligent asset can collect information about the processes themselves from one system and supply them to other systems. For example, permissions data from a DRM solution can be used to provide automated gatekeeping against inappropriate use of assets in the context of a production management solution. The assets, in effect, become the system integrators by using a builtin, and self-contained information collection and distribution system to pass relevant information from one domain to the next, from one part of the solution to the next.

\section{THE FUTURE HAS AN ACTIVE CONSTITUENCY NOW}

Some parts of this DAM-contributes-toworkflow concept are here today. XMP, active harvesting, standardized protocols, etc. This future already has the backing of managers who have a surplus of creativity and an iron mountain of storage but who now desperately 
need to make up the gap in managed productivity.

Such a concept may eventually evolve into a platform where the separate solutions today that solve particular business problems today are replaced by the workflow management solution that is capable of receiving and mediating all the cross-domain metadata. This solution will then truly turn information and metadata into knowledge that can leverage resources and streamline workflow across domains, and help people develop monitor and optimize end-toend workflows without overlap of effort and duplication of data. 